\title{
Patient with ST-elevation myocardial infarction, coronary artery embolism and no signs of coronary atherosclerosis in angiography
}

\author{
Adam Kern ${ }^{1,2}$, Robert Gil ${ }^{3,4}$, Jerzy Górny ${ }^{5}$, Ewa Sienkiewicz ${ }^{5}$, Krystian Bojko5${ }^{5}$ Grzegorz Wasilewski ${ }^{6}$ \\ ${ }^{1}$ Faculty of Medical Sciences, University of Varmia and Masuria, Olsztyn, Poland \\ ${ }^{2}$ Cardiac Catheterization Laboratory, Regional Specialist Hospital, Olsztyn, Poland \\ ${ }^{3}$ Invasive Cardiology Department, Central Clinical Hospital of the Internal Affairs and Administration Ministry, Warsaw, Poland \\ ${ }^{4}$ Institute of Experimental and Clinical Medicine, Polish Academy of Sciences, Warsaw, Poland \\ ${ }^{5}$ Department of Cardiology, Regional Specialist Hospital, Olsztyn, Poland \\ ${ }^{6}$ Department of Radiology and Medical Imaging, Regional Specialist Hospital, Olsztyn, Poland
}

Postep Kardiol Inter 2015; 11, 4 (42): 334-336

DOI: $10.5114 /$ pwki.2015.55607

\section{Case report}

A 43-year-old male patient with arterial hypertension and hypercholesterolemia, body mass index $39 \mathrm{~kg} / \mathrm{m}^{2}$, with no previous history of heart disease, was admitted due to sudden onset of chest pain $6 \mathrm{~h}$ prior to seeking medical help. ECG showed normal sinus rhythm with ST-segment elevation in leads aVR and V1-V3 and corresponding ST wave depression in leads I, II, III, aVL, and V5-V6. ST-elevation myocardial infarction (STEMI) was diagnosed in the patient.

Due to loud crescendo/decrescendo systolic murmur with punctum maximum at the aortic valve position, transthoracic echocardiography (TTE) was performed, confirming severe aortic stenosis (maximal pressure gradient $(\max P G)=91 \mathrm{~mm} \mathrm{Hg}$, mean $P G=65 \mathrm{~mm} \mathrm{Hg}$, aortic valve area $\left.(\mathrm{AVA})=0.6 \mathrm{~cm}^{2}\right)$. Furthermore, TTE showed apical akinesia. The left atrium size was moderately enlarged with diameter values of $4.0 \times 4.6 \mathrm{~cm}$.

After administration of $300 \mathrm{mg}$ of acetylsalicylic acid, $600 \mathrm{mg}$ of clopidogrel, and $100 \mathrm{mg}$ of enoxaparin, the patient was referred to the catheterization laboratory for primary percutaneous coronary intervention ( $\mathrm{pPCl}$ ). Coronary angiography showed no angiographic signs of atherosclerosis in coronary arteries, but the presence of thrombus in the mid segment of the left anterior descending artery (LAD) was detected (Figure 1).

Intracoronary bolus of eptifibatide $(18 \mathrm{mg}$ ) followed by intravenous infusion ( $12 \mathrm{mg} / \mathrm{h}$ ) was administered to the patient. Then coronary aspiration thrombectomy was performed in the LAD using a 6F Export AP Aspiration Catheter (Medtronic, Santa Clara, CA, USA). After successful thrombus removal, no residual stenosis was visible in the LAD (Figure 2). As there were no accompanying angiographic signs of atherosclerosis in other coronary arteries, we concluded that the thrombus was an embolus arising from a heart structure, most likely the left atrial appendage (LAA). Therefore no stent was implanted, the patient received optimal medical treatment, and further diagnostics to identify the potential source of the embolization were scheduled.

Transesophageal echocardiography carried out the next day confirmed severe aortic stenosis (tricuspid aortic valve), and no other heart defects (e.g. patent foramen ovale, atrial septal defect) as a potential mechanism of paradoxical embolism were detected. The left atrium was moderately enlarged with a thrombus-free LAA, but the presence of echogenic blood was confirmed. Short paroxysms of atrial fibrillation (AF) in 24-hour Holter ECG were observed.

After presentation to the Heart Team, the patient was scheduled for surgical aortic valve replacement. Until the heart surgery the patient was receiving enoxaparin S.C. (80 mg once a day). Clopidogrel (75 mg/day) was continued for 2 weeks. The patient was also treated with a $\beta$-blocker, ACE inhibitor and aspirin.

Four weeks later a mechanical aortic valve (Medtronic ATS 25 mm, Minneapolis, MN, USA) was successfully implanted. At discharge the patient received optimal medical treatment including a vitamin $\mathrm{K}$ antagonist.

\section{Corresponding author:}

Adam Kern MD, PhD, Faculty of Medical Sciences, University of Varmia and Masuria, 18 Żołnierska St, 10-457 Olsztyn, Poland, phone: +48 601659 213, fax: +48 895386 209, e-mail: adamkern@mail.com

Received: 21.01.2015, accepted: 23.03.2015. 


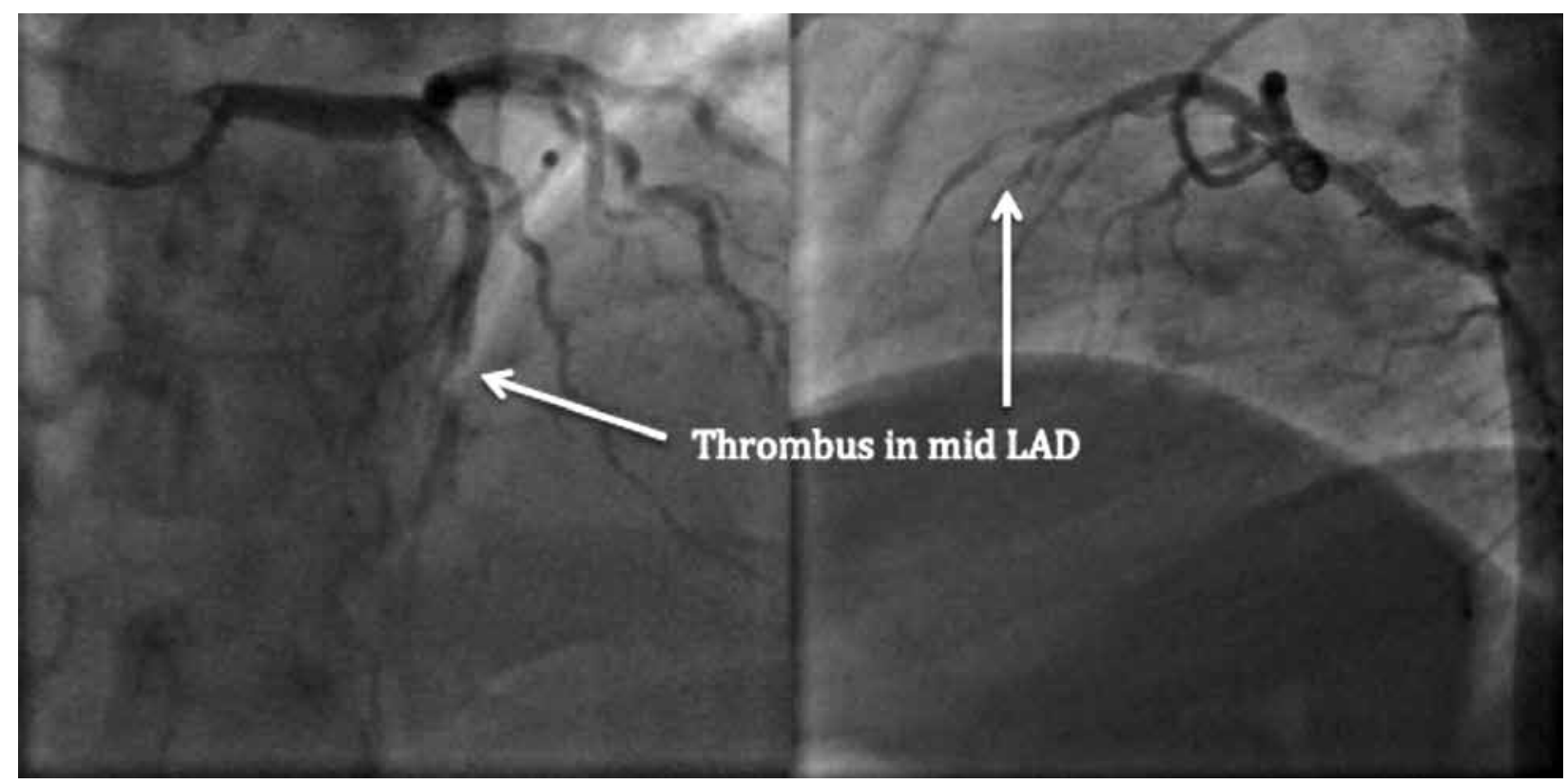

Figure 1. Thrombus present in the mid segment of the LAD

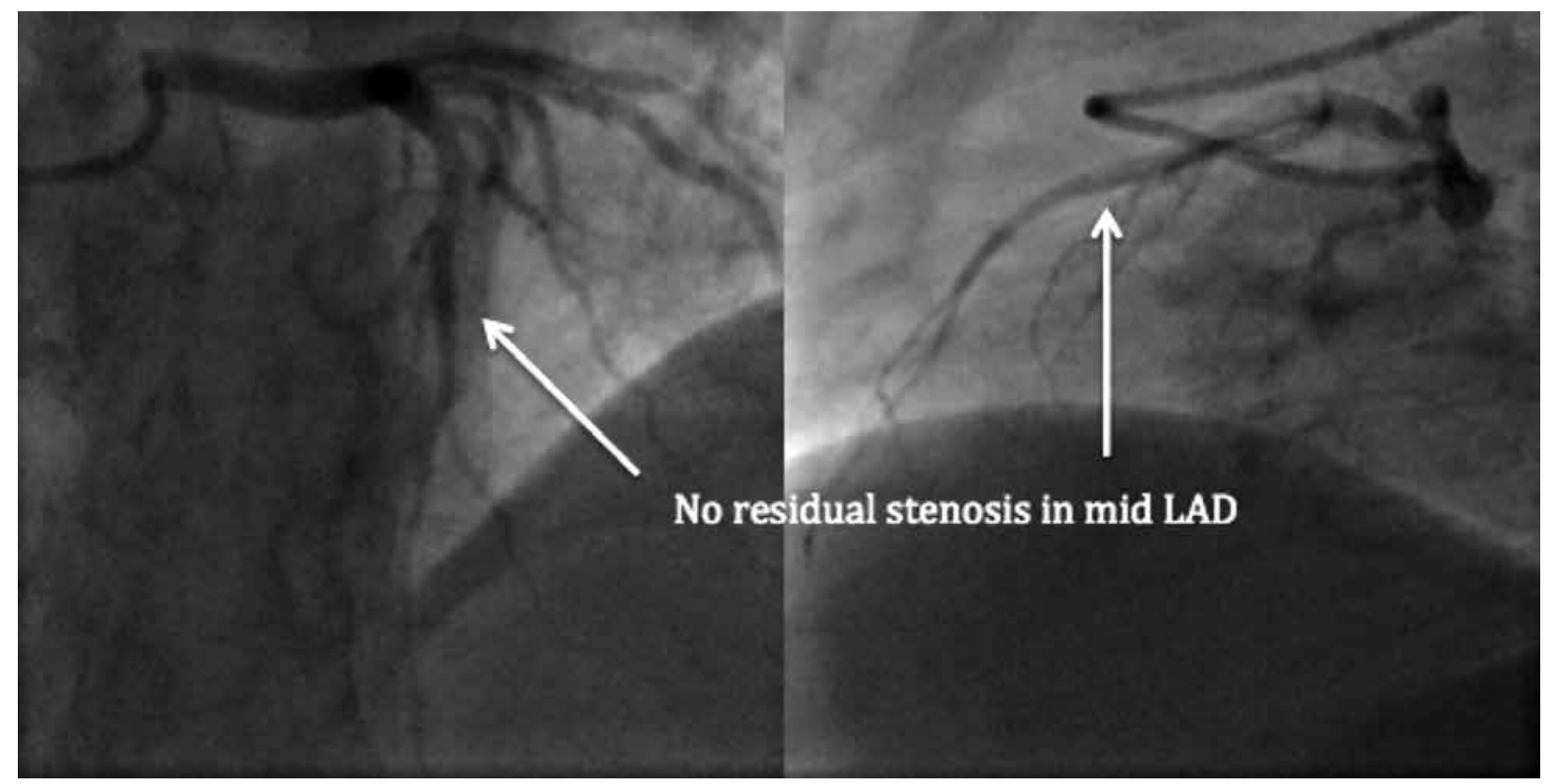

Figure 2. No residual stenosis visible in the LAD

In 1-year follow-up the patient was asymptomatic, in excellent clinical condition, with normal ECG and normal left ventricular function in TTE. Angiographic computed tomography confirmed the absence of CAD. Control 24hour Holter ECG showed no paroxysms of AF; however, the patient remains on oral anticoagulation due to the implanted artificial aortic valve.

\section{Discussion}

Coronary artery embolism is a rare cause of STEMI but remains a challenging diagnosis for the interven- tional cardiologist [1]. It represents an important clinical problem in terms of its etiology and clinical treatment [2], with incidence that varies from $4 \%$ up to $13 \%$ according to autopsy and coronary angiography studies [3, 4]. The prevalence of this nonatherosclerotic entity remains unknown due to difficulty in extensive diagnostics in the acute setting. Coronary embolism should always be suspected as a potential cause of STEMI in patients with no angiographic signs of coronary artery disease and the presence of AF, dilated cardiomyopathy, left ventricular aneurysm, prosthetic valves, infective endocarditis, tu- 
mors, valvular heart disease or known hypercoagulable states [4-8].

In such rare cases it is not easy to determine whether the thrombus arose in the coronary artery as a consequence of plaque rupture or erosion or was an embolus from another heart structure. The use of intravascular imaging such as intravascular ultrasound (IVUS) or optical coherence tomography (OCT) may be helpful to resolve the matter. In this particular case the angiographic images after successful thrombus aspiration and knowledge about thromboembolic risk factors led us to the conclusion that this was an embolic event and therefore IVUS was not used (OCT was not available at that moment). Angio CT images obtained one year after the procedure confirmed our speculations.

Previous cases have shown that most emboli involve the left coronary system, which could be due to the preferential flow into the artery related to aortic valve morphology [9]. Although in severe aortic stenosis the jet would more likely propel the embolus further into the aorta and less likely into the left coronary artery, this case shows that one should look for AF complications not only in the central nervous system but also in coronary arteries.

The urgent treatment of STEMI patients with an embolic cause is the same as in the case of atherosclerotic causes of STEMI and consists of aggressive antithrombotic medical treatment as well as primary $\mathrm{PCl}$ including thrombus aspiration [10] and administration of GP Ilb/ IIla antagonists. In cases with a large thrombus burden, systemic or intracoronary thrombolytic therapy may also be considered.

We assumed that in our case the embolus originated from the LAA. Occurrence of AF in 24-h ECG, valvular heart disease and obesity were risk factors for coronary embolism.

Stent implantation is not the optimal revascularization method when the underlying pathophysiological mechanism of STEMI is coronary embolism, especially when thrombus aspiration is successful and the vessel remains without any obstruction and/or signs of wall damage, which was the case in our patient. The clinical outcome of our patient proves that deferral of stent implantation was the right strategy.

Due to the low incidence of such cases there is no consensus on optimal antithrombotic treatment. Our plan was to keep the patient on dual-antiplatelet therapy for one month (as for plain old balloon angioplasty) using simultaneously low-molecular weight heparin s.c. (therapeutic dosage) until surgical valve replacement, which was scheduled 4 weeks after the pPCl. However, the cardiac surgeons stopped clopidogrel due to the risk of hemorrhagic complications, and luckily the preoperative period was complication-free. After surgery the patient was, according to current guidelines, given a vitamin $\mathrm{K}$ antagonist as chronic therapy.

\section{Conclusions}

Coronary artery thromboembolism as a nonatherosclerotic cause of STEMI is a rare finding with unknown prevalence. There is no consensus on the best treatment strategy for this group of patients. Intuitively the treatment should involve aggressive antithrombotic therapy and $\mathrm{pPCl}$ with aspiration thrombectomy. If possible, exclusion of a ruptured or eroded plaque using IVUS or preferably OCT should be considered. In cases of successful thromboaspiration, deferral of stent implantation supported by optimal medical treatment including shortterm use of antiplatelet agents and chronic oral anticoagulation (as stroke prevention) is crucial if the embolic nature of STEMI is confirmed.

\section{Conflict of interest}

The authors declare no conflict of interest.

\section{References}

1. Camaro C, Aengevaeren WRM. Acute myocardial infarction due to coronary artery embolism in a patient with atrial fibrillation. Neth Heart J 2009; 17: 297-9.

2. Garg RK, Jolly N. Acute myocardial infarction secondary to thromboembolism in a patient with atrial fibrillation. Int J Cardiol 2007; 123: 18-20.

3. Kiernan TJ, Flynn AMO, Kearney P. Coronary embolism causing myocardial infarction in a patient with mechanical aortic valve prosthesis. Int J Cardiol 2006; 112: 14-6.

4. Dogan M, Acikel S, Aksoy MMN, et al. Coronary saddle embolism causing myocardial infarction in a patient with mechanical mitral valve prosthesis: treatment with thrombolytic therapy. Int J Cardiol 2009; 135: 47-8.

5. Aslam MS, Sanghi V, Hersh S, et al. Coronary artery saddle embolus and myocardial infarction in a patient with prosthetic mitral valve. Catheter Cardiovasc Interv 2002; 57: 367-70.

6. Hung WC, Wu CJ, Chen WJ, et al. Transradial intracoronary catheter-aspiration embolectomy: for acute coronary embolism after mitral valve replacement. Tex Heart Inst J 2003; 30: 316-8.

7. Kraus PA, Lipman J. Coronary embolism causing myocardial infarction. Intensive Care Med 1990; 16: 215-6.

8. Kleczyński P, Dziewierz A, Legutko J, et al. Acute myocardial infarction in a patient with chronic renal failure and endocarditis. Kardiol Pol 2013; 71: 650-2.

9. Iwama T, Asami K, Kubo I, et al. Hypertrophic cardiomyopathy complicated with acute myocardial infarction due to coronary embolism. Int Med 1997; 36: 613-7.

10. Zasada W, Bartuś S, Królikowski T, et al. Patient with atrial fibrillation and myocardial infarction due to coronary artery embolism treated with thrombus aspiration. Kardiol Pol 2013; 71: 99-101. 